\title{
Arbeitssitzung über Gewässerverölung, Ölbekämpfung und Ölabbau: Eröffnungsansprache
}

\author{
WILFRIEd GUNKEL
}

Biologische Anstalt Helgoland, Meeresstation, Helgoland

\section{Meine Herren!}

Im Auftrage des Leitenden Direktors der Biologischen Anstalt Helgoland, Herrn Professor Dr. O. KINNE, heiße ich Sie herzlich willkommen in unserer Meeresstation. Ich freue mich, daß Sie in so großer Zahl unserer Einladung zu einem Arbeitstreffen von Spezialisten Folge geleistet haben. Schon in dem soeben beendeten Internationalen Symposion unserer Anstalt über „Biologische und bydrographische Probleme der Wasserverunreinigung in der Nordsee und angrenzenden Gewässern" sind Vorträge auch zum Thema der Gewässerverölung gehalten worden.

Derartige große Tagungen sind für den Gedankenaustausch und die persönlichen Kontakte $z$ wischen Wissenschaftlern notwendig und ertragreich. Sie können aber in der heutigen Zeit mit ihrer explosionsartigen Vermehrung des wissenschaftlichen Datenmaterials und divergierender Spezialisierung nicht die alleinige Form des Erfahrungsaustausches sein. Häufig werden auf diesen Symposien abgeschlossene Arbeiten vorgetragen, deren Inhalt und Ergebnisse in der Diskussion zwangsläufig „verteidigt" werden müssen. Werden nicht oft die schwachen Stellen der Untersuchungen und der Methodik kaum erwähnt? Kommen nicht gutgemeinte Anregungen häufig zu spät, da die Arbeit ja abgeschlossen ist? Darüber hinaus bringen sehr große Kongresse mit ihren meist umfangreichen Programmen es mit sich, daß nur eine recht begrenzte Zeit für die Diskussionen zur Verfügung steht; sie bleiben dabei vielfach an der Oberfläche. Häufig sind daher die Vortragspausen der ertragreichste Teil eines großen Symposions. Hier werden Kontakte geknüptt und im persönlichen Gespräch tiefer gehende Diskussionen geführt, die jedoch den meisten Teilnehmern entgehen. Einen Ausweg bieten Arbeitssitzungen im kleinen Kreis. So haben sich im Sommer 1964 elf Gewässermikrobiologen in der Meeresstation der Biologischen Anstalt Helgoland auf Helgoland zu einer offenen und teilweise harten Diskussion zusammengefunden (Helgoländer wiss. Meeresunters. 11, 327-340). Wir haben ohne Ansehen der Person frei diskutiert, und mehrere Kollegen haben die von ihnen verwandten Methoden demonstriert. Wir kamen am Ende der Arbeitssitzung $1964 \mathrm{zu}$ der Ansicht, daß uns dieses Treffen sehr viel gegeben hat. Weitere gewässermikrobiologische Arbeitstreffen wurden später in Plön, Bremerhaven und Kiel abgehalten.

In diesem Zusammenhang sollte auch das "Interdisciplinary Communications Program" der New Yorker Academy of Sciences Erwähnung finden. Seit Jahren organi- 
siert diese Akademie Treffen mit einer begrenzten Anzahl von Teilnehmern. Hier werden Themen behandelt, die verschiedene Disziplinen berühren. So trafen wir uns zum Beispiel im Januar 1966 in Princeton, um das Thema „Some unresolved questions in marine microbiology" eingehend zu diskutieren. Das Schwergewicht bei diesen Treffen liegt auf den Diskussionen. Diese werden in vollem Umfang veröffentlicht, so daß der Leser an der Lebendigkeit der Erörterungen teilnehmen kann. Auch die Diskussionen dieses Arbeitstreffens sollen publiziert werden. Die Diskussion gibt uns die Möglichkeit, auch Einzelergebnisse zu erörtern, deren Veröffentlichung als eigenständige Publikation nicht gerechtfertigt ist, die jedoch den Kollegen nützliche Anregungen vermitteln können.

Die Idee zu diesem Treffen wurde geboren, als zur Zeit des Tankerunfalls der "Anne Mildred Brøvig" Professor MANN, Dr. KüHL, Dr. VAuk und ich auf Helgoland zusammentrafen, und wir über den Ablauf der Verölung und die angewandten Bekämpfungsmethoden sprachen. Wir kamen zu der Ansicht, daß sich die Fachleute, die von den verschiedensten Arbeitsrichtungen herkommend an Problemen über Gewässerverölung, Olbekämpfung und Olabbau interessiert sind, zu einem Erfahrungsaustausch zusammenfinden sollten. Wir dachten an Meeresbiologen, Ornithologen, Chemiker, Mikrobiologen, Emulgatorfachleute, Vertreter der Olindustrie, Kollegen von der Gewässeraufsicht bzw. Überwachung sowie Praktiker der Wasser- und Schiffahrtsämter.

Ich freue mich sehr, daß Sie der Einladung gefolgt sind, und daß wir heute Fachleute aller dieser Richtungen einschließlich eines Vertreters des Bundesministeriums für Gesundheitswesen unter uns haben. Hierdurch ist ein Gedankenaustausch auf breiter, interdisziplinärer Basis möglich.

Einige der Kollegen kennen sich bereits. Das ist jedoch nicht allgemein der Fall, so daß ich vorschlagen würde, daß wir uns miteinander bekannt machen, indem jeder einzelne in wenigen Sätzen mitteilt, worüber er arbeitet.

Im Anschluß an diese Vorstellung werden wir 6 Vorträge hören und spezielle Probleme der Gewässerverölung in zwanglosen Diskussionen eingehend erörtern. 